\title{
Automates et algébricités
}

\author{
par JEAN-PAUL ALLOUCHE
}

\begin{abstract}
RÉSUMÉ. Dans quelle mesure la régularité des chiffres d'un nombre réel dans une base entière, celle des quotients partiels du développement en fraction continuée d'un nombre réel, ou celle des coefficients d'une série formelle sont-elles liées à l'algébricité ou à la transcendance de ce réel ou de cette série formelle? Nous proposons un survol de résultats récents dans le cas où la régularité évoquée ci-dessus est celle de suites automatiques, substitutives, ou sturmiennes.
\end{abstract}

ABstract. How related are the following: the regularity of the digits of a real number in an integer base, the regularity of the partial quotients of the continued fraction expansion of a real number, or the regularity of the coefficients of a formal power series, and the algebraicity or transcendence of this real number or of this formal power series? We give a survey of recent results for regularity properties of automatic, substitutive, or Sturmian sequences.

\section{Introduction (English)}

Given a sequence with values in a finite set $\mathcal{A}$, say $\mathcal{A}=\{0,1\}$, is this sequence algebraic or transcendental? This might depend on the interpretation of the sequence. More precisely, let $\left(u_{n}\right)_{n \geq 0}$ be a binary sequence, the following questions can be asked.

- Is the real number $\sum_{n \geq 0} u_{n} / 2^{n}=u_{0} \cdot u_{1} \cdots u_{n} \cdots$ transcendental over the rationals? What happens if 2 is replaced by another base of numeration $b \geq 2$ ? What if $b$ is a noninteger base?

- Is the formal power series $\sum_{n \geq 0} u_{n} X^{n}$ transcendental over the field $\mathbb{Q}(X)$ of rational functions on $\mathbb{Q}$ ?

- Is the formal power series $\sum_{n \geq 0}\left(u_{n} \bmod p\right) X^{n}$ transcendental over the field $\mathbb{F}_{p}(X)$, where $p$ is a prime number? Is the result the same if $p=2$ and if $p=3$ ?

- Replacing $\left(u_{n}\right)_{n \geq 0}$ by $\left(1+u_{n}\right)_{n \geq 0}$, say, is the real number with continued fraction expansion $\left[1+u_{0}, 1+u_{1}, \cdots, 1+u_{n}, \cdots\right]$ a transcendental number? 
One might think that "irregularities" or "regularities" of the sequence $\left(u_{n}\right)_{n \geq 0}$ play a rôle in the answers to the questions above. We will use two classical sequences, the Thue-Morse sequence and the Fibonacci sequence, as a leading thread to explore some of the questions above. What follows is in French, but probably easy to read for an English speaker: references in the bibliography, including Chapters 12 and 13 of [6] will provide the reader with more details.

\section{Introduction}

Peut-on trouver des régularités dans le développement décimal du réel $\sqrt{2}=1,4142 \cdots$ ? Une conjecture (voir [8]) qui semble hors d'atteinte est que la réponse à cette question est non : plus précisément cette conjecture stipule que chaque chiffre entre 0 et 9 apparait avec la même fréquence $1 / 10$, que chaque couple de chiffres $00,01, \ldots, 99$ apparait avec la fréquence $1 / 100, \ldots$, que chaque bloc de $k$ chiffres apparaît avec la fréquence $1 / 10^{k}$. Un nombre ayant cette propriété - qui dit essentiellement que ses chiffres en base 10 sont répartis "au hasard" - est dit normal en base 10. La conjecture à laquelle nous faisons allusion ci-dessus est que tout nombre algébrique irrationnel est normal en toute base entière.

À rebours un nombre réel dont les chiffres "ne sont pas assez au hasard" devrait être rationnel ou transcendant. L'objet de ce survol est de partir de la célèbre suite de Prouhet-Thue-Morse

$$
01101001100101101 \cdots \text {, }
$$

de considérer différentes interprétations de cette suite (chiffres d'un nombre réel en base 10 , en base $2, \ldots$, coefficients d'une série formelle ou entière sur le corps $\mathbb{Q}$ des nombres rationnels, coefficients d'une série formelle sur un corps fini, quotients partiels d'une fraction continuée, ...), et de (tenter de) répondre pour chacune de ces interprétations à la question de savoir si l'objet correspondant est algébrique ou non, puis d'indiquer les généralisations possibles. Nous allons voir que la nature arithmétique (algébricité ou transcendance) d'une suite dépend de l'interprétation que l'on en donne (d'où le "s" au mot "algébricités" dans le titre), et nous indiquerons plusieurs références à travers lesquelles le lecteur pourra continuer cette réflexion.

Notons pour clore cette introduction qu'il sera, entre autres, question de suites automatiques : l'auteur se souvient avoir donné des propriétés de ces suites dans un exposé aux Journées Arithmétiques de Besançon en 1985, et le lecteur sera peut-être amusé de savoir que ces suites sont maintenant le sujet de la rubrique 11B85 dans la classification des Mathematical Reviews, toute ressemblance entre ces deux occurrences du nombre " 85 " étant bien sûr tout à fait fortuite. 


\section{Les suites de Prouhet-Thue-Morse et de Fibonacci}

3.1. La suite de Prouhet-Thue-Morse. La suite $\left(u_{n}\right)_{n \geq 0}$ de ProuhetThue-Morse est définie par

$$
u_{n}= \begin{cases}0 & \text { si le développement binaire de } n \text { a un nombre pair de } 1, \\ 1 & \text { si le développement binaire de } n \text { a un nombre impair de } 1\end{cases}
$$

On peut aussi définir cette suite par le procédé "mécanique" suivant appelé morphisme, substitution ou règle d'inflation :

$$
\begin{aligned}
& 0 \rightarrow 01 \\
& 1 \rightarrow 10
\end{aligned}
$$

Cette règle définit un morphisme du monoïde libre $\{0,1\}^{*}$, c'est-à-dire de l'ensemble des mots sur l'alphabet $\{0,1\}$ muni de l'opération de concaténation. Par exemple

$$
011100 \rightarrow(01)(10)(10)(10)(01)(01)=011010100101 .
$$

L'itération de ce morphisme en partant de 0 donne successivement

0

01

0110

011010001

d'où, à la limite, une suite infinie

$$
01101001100101101 \cdots,
$$

appelée suite de Prouhet-Thue-Morse, qui est par construction, point fixe de ce morphisme étendu aux suites infinies. Le lecteur pourra consulter $[18,21,25,26,5]$. Notons que la construction même de cette suite montre qu'elle n'est pas "au hasard".

3.2. La suite de Fibonacci. La suite $\left(v_{n}\right)_{n \geq 0}$ de Fibonacci est définie comme le point fixe du morphisme

$$
\begin{aligned}
& 0 \rightarrow 01 \\
& 1 \rightarrow 0 .
\end{aligned}
$$

En partant de 0 et en itérant ce morphisme comme ci-dessus, on obtient

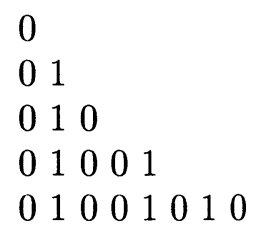


d'où à la limite

$$
01001010010010 \cdots
$$

On notera que le morphisme qui engendre la suite de Fibonacci n'est pas de longueur constante : les images de 0 et de 1 n'ont pas le même nombre de lettres. Ceci se traduit en particulier par le fait que le terme général $v_{n}$ de la suite de Fibonacci ne peut pas être calculé "aisément" en fonction du développement binaire de $n$ comme c'était le cas pour le terme général $u_{n}$ de la suite de Prouhet-Thue-Morse. Le lecteur montrera en exercice que $v_{n}$ vaut 0 ou 1 suivant que le dernier chiffre dans le développement de $n$ en base de Fibonacci (c'est-à-dire dans la base $1,2,3,5,8,13, \ldots$ ) vaut lui-même 0 ou 1.

\section{Nombres réels associés}

Une première interprétation de la suite de Prouhet-Thue-Morse est obtenue en insérant une virgule :

$$
0,1101001100101101 \cdots
$$

Cette écriture peut être alors considérée comme le développement d'un nombre réel en base 2 (ou en base 3 , ou en base 10 ...). Un théorème dû à Mahler [17] affirme que tous ces nombres sont transcendants.

Théorème 4.1 (Mahler). Soit $\left(u_{n}\right)_{n \geq 0}$ la suite de Prouhet-Thue-Morse. Soit $b$ un nombre entier $\geq 2$. Alors le nombre réel

$$
\sum_{n=0}^{\infty} \frac{u_{n}}{b^{n}}
$$

est transcendant sur $\mathbb{Q}$.

Idée de la démonstration. Mahler considère la fonction $F$ définie pour les $X$ complexes tels que $|X|<1$ par $F(X)=\prod_{n=0}^{\infty}\left(1-X^{2^{n}}\right)$. Cette fonction satisfait clairement à l'équation fonctionnelle $F(X)=(1-X) F\left(X^{2}\right)$. Mahler montre que, si $\alpha$ est un nombre algébrique dont le module vérifie $0<|\alpha|<1$, alors $F(\alpha)$ est transcendant sur $\mathbb{Q}$ : l'idée de la preuve consiste à remplacer dans une éventuelle équation algébrique vérifiée par $F(\alpha)$ cette valeur par $(1-\alpha) F\left(\alpha^{2}\right)$ et à itérer pour obtenir une contradiction (ceci n'est bien sûr qu'une esquisse).

On note alors que $F(X)=\sum_{n=0}^{\infty}(-1)^{u_{n}} X^{n}$ (utiliser la propriété de tout entier $n$ d'avoir une écriture unique comme somme de puissances de 2 , c'est-à-dire en base 2). Puis on constate que

$$
F(X)=\sum_{n=0}^{\infty}(-1)^{u_{n}} X^{n}=\sum_{n=0}^{\infty}\left(1-2 u_{n}\right) X^{n}=\frac{1}{1-X}-2 \sum_{n=0}^{\infty} u_{n} X^{n}
$$


(utiliser le fait que $u_{n}$ ne prend que les valeurs 0 et 1 ), donc

$$
\sum_{n=0}^{\infty} \frac{u_{n}}{b^{n}}=\frac{1}{2}\left(\frac{b}{b-1}-F\left(\frac{1}{b}\right)\right)
$$

est transcendant sur $\mathbb{Q}$ pour $b$ entier $\geq 2$.

Un théorème analogue est vrai pour la suite de Fibonacci. Il semble que ce soit Danilov [13] qui l'ait démontré pour la première fois.

Théorème 4.2 (Danilov). Soit $\left(v_{n}\right)_{n \geq 0}$ la suite de Fibonacci. Soit $b$ un nombre entier supérieur ou égal à 2. Alors le nombre réel

$$
\sum_{n=0}^{\infty} \frac{v_{n}}{b^{n}}
$$

est transcendant.

Remarque. Les théorèmes 4.1 et 4.2 ci-dessus peuvent être généralisés de deux manières : d'une part remplacer le morphisme qui engendre la suite de Prouhet-Thue-Morse ou celle de Fibonacci par un morphisme satisfaisant à certaines conditions (voir $[15,7,19]$ ), d'autre part remplacer la suite de Fibonacci par n'importe quelle suite sturmienne, c'est-à-dire qui code avec des 0 et des 1 la trajectoire d'une boule sur un billard carré lorsque l'angle d'attaque a une pente irrationnelle (voir [15] et les références données dans [3], voir aussi [2]). Notons que les généralisations dans [15] et [7] ne reposent pas comme dans le théorème 4.1 sur l'existence d'une équation fonctionnelle, mais sur le fait qu'une propriété combinatoire d'une suite, en l'occurrence contenir des "presque cubes" arbitrairement longs pas "trop loin" du début de la suite, se traduit en propriété d'approximation par des rationnels du réel associé : ces approximations sont trop "bonnes" pour que le nombre approché puisse être algébrique irrationnel (voir les détails dans [15] ou dans le survol [3]).

Remarque. On peut se demander ce qui se passe pour des bases non entières. Prenons par exemple le nombre d'or $\tau=(1+\sqrt{5}) / 2$. Une conséquence du résultat de Mahler donné dans la preuve du théorème 4.1 ci-dessus est que, en appelant toujours $\left(u_{n}\right)_{n \geq 0}$ la suite de Prouhet-ThueMorse, le nombre $\sum_{n=0}^{\infty} \frac{u_{n}}{\tau^{n}}$ est transcendant car $\tau$ est algébrique. La transcendance du réel $\sum_{n=0}^{\infty} \frac{v_{n}}{\tau^{n}}$, où $\left(v_{n}\right)_{n \geq 0}$ est la suite de Fibonacci, est démontrée dans [17] ; pour des généralisations $\left(\left(v_{n}\right)_{n \geq 0}\right.$ remplacée par certaines suites sturmiennes, $\tau$ remplacé par un nombre algébrique ou seulement un Pisot ou un Salem) voir $[17,16,1]$. 


\section{Séries associées sur le corps des nombres rationnels}

Comme indiqué dans l'introduction on peut considérer les suites $\left(u_{n}\right)_{n \geq 0}$ et $\left(v_{n}\right)_{n \geq 0}$ comme les coefficients de séries formelles ou entières sur $\mathbb{Q}$ et se demander si ces séries sont algébriques, c'est-à-dire algébriques sur le corps $\mathbb{Q}(X)$ des fractions rationnelles à coefficients rationnels.

Théorème 5.1. Les deux séries (entières ou formelles) $\sum_{n=0}^{\infty} u_{n} X^{n}$ et $\sum_{n=0}^{\infty} v_{n} X^{n}$ sont transcendantes sur $\mathbb{Q}(X)$.

Démonstration. La preuve consiste seulement à remarquer que la suite de Prouhet-Thue-Morse et celle de Fibonacci sont à valeurs dans un ensemble fini, et ne sont ni l'une ni l'autre ultimement périodiques (pour la première c'est par exemple une conséquence du résultat de Thue qui stipule en particulier qu'elle ne contient aucun cube, c'est-à-dire aucun bloc répété trois fois consécutivement, pour la seconde c'est par exemple une conséquence du fait que la fréquence des 0 dans cette suite existe et est égale à $(\sqrt{5}-1) / 2$ donc irrationnelle). On en déduit que les séries $\sum_{n=0}^{\infty} u_{n} X^{n}$ et $\sum_{n=0}^{\infty} v_{n} X^{n}$ sont irrationnelles. On applique alors un théorème dû à Fatou [14, p. 368] : si une série entière est à coefficients entiers et si son rayon de convergence est égal à 1 , alors elle est soit rationnelle, soit transcendante. (Le théorème de Pólya-Carlson donne plus de précisions sur les singularités d'une telle série, voir [20].)

\section{Remarque.}

- Le lecteur pourrait se demander pourquoi on ne démontre pas la transcendance de la série entière $\sum_{n=0}^{\infty} u_{n} X^{n}$ par exemple, en utilisant le fait que sa valeur pour $X=1 / 2$ est transcendante d'après le théorème 4.1 : c'est que la preuve de ce théorème utilise en fait déjà la transcendance de la série entière.

- Le résultat de ce paragraphe peut bien sûr être étendu aux suites engendrées par morphismes ou aux suites sturmiennes, lorsqu'elles ne sont pas ultimement périodiques.

\section{Séries associées sur un corps fini}

On peut aussi considérer les suites $\left(u_{n}\right)_{n \geq 0}$ et $\left(v_{n}\right)_{n \geq 0}$ ci-dessus comme les coefficients de séries formelles sur le corps fini à $q$ éléments $\mathbb{F}_{q}\left(q=p^{a}\right.$ où $p$ est premier égal à la caractéristique du corps) et se demander si ces séries sont algébriques, c'est-à-dire algébriques sur le corps $\mathbb{F}_{q}(X)$ des fractions rationnelles à coefficients dans $\mathbb{F}_{q}$. Ici nous allons voir que, pour la suite $\left(u_{n}\right)_{n \geq 0}$, les choses diffèrent suivant que $q$ est une puissance de 2 ou pas.

\section{Théorème 6.1.}

- La série formelle $\sum_{n=0}^{\infty} u_{n} X^{n}$ est algébrique sur le corps $\mathbb{F}_{q}(X)$ si et seulement si $q$ est une puissance de 2 . 
- La série formelle $\sum_{n=0}^{\infty} v_{n} X^{n}$ est transcendante sur le corps $\mathbb{F}_{q}(X)$ pour tout entier $q \geq 2$.

Démonstration. La série formelle $\sum_{n=0}^{\infty} u_{n} X^{n}$ est algébrique sur le corps $\mathbb{F}_{q}(X)$ lorsque $q$ est une puissance de 2 : il suffit de le montrer pour $q=2$. Or, on a facilement sur $\mathbb{F}_{2}$ les relations

$$
\forall n \geq 0, \quad u_{2 n}=u_{n} \text { et } u_{2 n+1}=1+u_{n},
$$

d'où, si l'on note $G=G(X)=\sum_{n=0}^{\infty} u_{n} X^{n}$,

$$
\begin{aligned}
G & =\sum_{n=0}^{\infty} u_{2 n} X^{2 n}+\sum_{n=0}^{\infty} u_{2 n+1} X^{2 n+1}=\sum_{n=0}^{\infty} u_{n} X^{2 n}+\sum_{n=0}^{\infty}\left(1+u_{n}\right) X^{2 n+1} \\
& =(1+X)\left(\sum_{n=0}^{\infty} u_{n} X^{2 n}\right)+\frac{X}{1-X^{2}} \\
& =(1+X)\left(\sum_{n=0}^{\infty}\left(u_{n}\right)^{2} X^{2 n}\right)+\frac{X}{1-X^{2}}
\end{aligned}
$$

On se souvient alors que l'on travaille modulo 2 , donc que $(a+b)^{2}=a^{2}+b^{2}$ et que $-1=+1$, d'où

$$
G=(1+X)\left(\sum_{n=0}^{\infty} u_{n} X^{n}\right)^{2}+\frac{X}{(1+X)^{2}}=(1+X) G^{2}+\frac{X}{(1+X)^{2}} .
$$

En d'autres termes $G$ satisfait à l'équation $(1+X)^{3} G^{2}+(1+X)^{2} G+X=0$, et est donc algébrique sur $\mathbb{F}_{2}(X)$.

D'après la définition de la suite $\left(u_{n}\right)_{n \geq 0}$ comme point fixe d'un morphisme donnée plus haut, cette suite est 2-automatique (au sens de Cobham [12]). Cette suite, qui n'est pas ultimement périodique comme déjà indiqué, ne peut donc pas être $k$-automatique lorsque $k$ n'est pas une puissance de 2 (d'après le théorème fondamental de Cobham dans [11]). La série $\sum_{n=0}^{\infty} u_{n} X^{n}$ considérée cette fois comme à coefficients dans le corps $\mathbb{F}_{q}(X)$ ne peut donc pas être algébrique si $q$ n'est pas une puissance de 2 , en vertu du théorème de Christol $[9,10]$.

Le cas de la série $\sum_{n=0}^{\infty} v_{n} X^{n}$ est plus simple : si cette série était algébrique sur le corps $\mathbb{F}_{q}(X)$ pour un entier $q \geq 2$, alors la suite $\left(v_{n}\right)_{n \geq 0}$ serait $q$-automatique $[9,10]$. Or un théorème de Cobham [12] stipule que, si la fréquence d'une lettre qui apparaît dans une suite automatique existe, elle doit être un nombre rationnel. Comme nous l'avons dit plus haut, ce n'est pas le cas pour la fréquence de la lettre 0 dans la suite $\left(v_{n}\right)_{n \geq 0}$, qui existe et vaut $(\sqrt{5}-1) / 2$. 
Remarque. L'outil principal de ce paragraphe est le théorème de Christol [9], voir aussi l'article de Christol, Kamae, Mendès France et Rauzy [10], dont voici un énoncé. Soit $\left(w_{n}\right)_{n \geq 0}$ une suite à valeurs dans un ensemble fini $\mathcal{A}$. Soit $p$ un nombre premier. La suite $\left(w_{n}\right)_{n \geq 0}$ est p-automatique, c'est-à-dire image lettre à lettre d'un point fixe d'un morphisme de longueur $p$ sur un ensemble fini $\mathcal{B}$, si et seulement s'il existe un entier $a \geq 1$ et une injection $\iota$ de $\mathcal{A}$ dans le corps fini $\mathbb{F}_{p^{a}}$ tels que la série formelle $\sum_{n=0}^{\infty} \iota\left(w_{n}\right) X^{n}$ soit algébrique sur $\mathbb{F}_{p^{a}}(X)$.

\section{Fractions continuées associées}

Nous avons vu plusieurs "interprétations" des suites de Prouhet-ThueMorse et de Fibonacci : comme développement d'un nombre réel dans une base donnée, comme série formelle ou entière sur les rationnels, comme série formelle sur un corps fini. On peut aussi considérer ces suites comme les quotients partiels d'une fraction continuée. À vrai dire, comme des quotients partiels sont des entiers strictement positifs (sauf peut-être le premier), nous allons translater nos deux suites et définir :

$$
\begin{aligned}
& \left(u_{n}^{\prime}\right)_{n \geq 0}=\left(1+u_{n}\right)_{n \geq 0}=12212112211212212 \cdots \text {, } \\
& \left(v_{n}^{\prime}\right)_{n \geq 0}=\left(1+v_{n}\right)_{n \geq 0}=12112121121121 \cdots
\end{aligned}
$$

Tout ce qui a été dit dans les paragraphes précédents sur les propriétés respectives des différentes interprétations des suites $u$ et $v$ reste vrai si on les remplace respectivement par les suites $u^{\prime}$ et $v^{\prime}$. Nous nous intéressons maintenant aux fractions continuées associées, par exemple

$$
\left[0, u_{0}^{\prime}, u_{1}^{\prime}, u_{2}^{\prime}, \cdots\right]:=\frac{1}{u_{0}^{\prime}+\frac{1}{u_{1}^{\prime}+\frac{1}{u_{2}^{\prime}+\ldots}}}
$$

Le premier résultat que nous citons est dû à Queffélec [22].

Théorème 7.1 (Queffélec). Soit $\left(u_{n}^{\prime}\right)_{n \geq 0}$ la translatée de la suite de Prouhet-Thue-Morse ci-dessus. Alors le nombre réel $x:=\left[0, u_{0}^{\prime}, u_{1}^{\prime}, u_{2}^{\prime}, \cdots\right]$, est transcendant. Plus généralement, soient a et $b$ deux entiers strictement positifs distincts, et soit $\left(z_{n}\right)_{n \geq 0}$ la suite définie par $z_{n}=a$ si $u_{n}=0$ et $z_{n}=b$ si $u_{n}=1$, où $\left(u_{n}\right)_{n \geq 0}$ est la suite de Prouhet-Thue-Morse. Alors le nombre réel $y:=\left[0, z_{0}, z_{1}, z_{2}, \cdots\right]$ est transcendant.

Remarque. La méthode utilisée pour prouver ce résultat relève un peu de la même "philosophie" que ce que nous indiquons à la fin de la remarque qui suit le théorème $4.2:$ si la suite des quotients partiels d'un nombre réel à quotients partiels bornés contient des "presque carrés" arbitrairement longs au début, alors le réel correspondant est "trop bien approché" par des nombres quadratiques et il est donc quadratique ou transcendant. On pourra consulter [22] ou le survol [3] pour en savoir plus. 
On peut généraliser le théorème 7.1 ci-dessus à d'autres points fixes de morphismes [4]. On peut aussi utiliser la même technique pour les suites sturmiennes [4].

Théorème 7.2 (Allouche, Davison, Queffélec, Zamboni). Soient $a$ et $b$ deux nombres entiers strictement positifs distincts. Soit $\left(t_{n}\right)_{n \geq 0}$ la suite obtenue dans une suite sturmienne quelconque en remplaçant les 0 par des $a$ et les 1 par des $b$. Alors le nombre réel $t:=\left[0, t_{0}, t_{1}, t_{2}, \cdots\right]$ est transcendant.

Remarque. Le lecteur trouvera une jolie occurrence de la fraction continuée dont la suite des quotients partiels $\left(t_{n}\right)_{n \geq 0}$ est la suite de Fibonacci sur l'alphabet $\{1,2\}$ dans le cadre de l'approximation simultanée d'un nombre et de son carré ou de l'approximation d'un nombre réel par des entiers algébriques cubiques dans $[23,24]$.

\section{Conclusion}

Le caractère algébrique ou transcendant d'une suite dépend donc bien sûr de l' "interprétation" de cette suite, et les lecteurs imagineront sans doute d'autres interprétations que celles données ci-dessus. Ces dernières, brièvement évoquées dans ce survol, donnent lieu à de nombreuses questions ouvertes même si on se limite à des suites automatiques : on trouvera plus de résultats, mais aussi plus de questions, dans les chapitres 12 et 13 de [6].

\section{Remerciements}

L'auteur remercie chaleureusement le comité scientifique et les organisateurs des Journées Arithmétiques 2003 à Graz. Il tient aussi à remercier B. Adamczewski et $\mathrm{V}$. Berthé pour leur lecture attentive d'une précédente version de ce texte et leurs remarques et suggestions.

Note ajoutée aux épreuves (décembre 2004).

$\mathrm{B}$. Adamczewski, Y. Bugeaud et $\mathrm{F}$. Luca ont obtenu le très joli résultat général suivant via le théorème du sous-espace de Schmidt : un nombre réel irrationnel dont le développement dans une base entière contient $O(n)$ blocs de longueur $n$ est nécessairement transcendant. Ce théorème implique en particulier que tout nombre irrationnel à développement automatique (comme le nombre de Thue-Morse) ou à développement sturmien (comme le nombre de Fibonacci) est transcendant. Voir :

B. Adamczewski, Y. Bugeaud, F. Luca, Sur la complexité des nombres algébriques. C. R. Math. Acad. Sci. Paris 339 (2004), 11-14.

B. Adamczewski, Y. Bugeaud, On the complexity of algebraic numbers. Soumis, (2004). 


\section{Références}

[1] B. Adamczewsi, Transcendance de nombres réels et p-adiques par la méthode de Roth. Prétirage, 2004.

[2] B. AdAmczewsi, J. CASSAigne, On the transcendence of real numbers with a regular expansion. J. Number Theory 103 (2003), 27-37.

[3] J.-P. Allouche, Nouveaux résultats de transcendance de réels à développement non aléatoire. Gaz. Math. 84 (2000), 19-34.

[4] J.-P. Allouche, J. L. Davison, M. Queffélec, L. Q. Zamboni, Transcendence of Sturmian or morphic continued fractions. J. Number Theory 91 (2001), 39-66.

[5] J.-P. Allouche, J. Shallit, The ubiquitous Prouhet-Thue-Morse sequence, in Sequences and their applications, Proceedings of SETA'98, C. Ding, T. Helleseth and H. Niederreiter (Eds.). Springer, 1999, pp. 1-16.

[6] J.-P. Allouche, J. Shallit, Automatic sequences. Theory, Applications, Generalizations. Cambridge University Press, 2003, xvi + 571 pages.

[7] J.-P. Allouche, L. Q. ZAmBoni, Algebraic irrational binary numbers cannot be fixed points of non-trivial constant length or primitive morphisms. J. Number Theory 69 (1998), 119124.

[8] É. BoREL, Sur les chiffres décimaux de $\sqrt{2}$ et divers problèmes de probabilités en châ̂ne. C. R. Acad. Sci. Paris 230 (1950), 591-593. Réédité dans : Euvres d'É. Borel, vol. 2. Éditions du CNRS, Paris, 1972, pp. 1203-1204.

[9] G. Christol, Ensembles presque périodiques $k$-reconnaissables. Theoret. Comput. Sci. 9 (1979), 141-145.

[10] G. Christol, T. Kamae, M. Mendès France, G. Rauzy, Suites algébriques, automates et substitutions. Bull. Soc. Math. France 108 (1980), 401-419.

[11] A. Совнам, On the base-dependence of sets of numbers recognizable by finite automata. Math. Systems Theory 3 (1969), 186-192.

[12] A. Coвham, Uniform tag sequences. Math. Systems Theory 6 (1972), 164-192.

[13] L. V. DANILOV, Some classes of transcendental numbers (en russe). Mat. Zametki 12 (1972), 149-154. Traduit dans : Math. Notes Acad. Sci. USSR 12 (1972), 524-527.

[14] P. Fatou, Séries trigonométriques et séries de Taylor. Acta Math 30 (1906), 335-400.

[15] S. FERENCZI, C. MAUduIT, Transcendence of numbers with a low complexity expansion. J. Number Theory 67 (1997), 146-161.

[16] J. H. Loxton, A. J. vAN DER POORTEN, Arithmetic properties of certain functions in several variables, III. Bull. Austral. Math. Soc. 16 (1977), 15-47.

[17] K. MAHLER, Arithmetische Eigenschaften der Lösungen einer Klasse von Funktionalgleichungen. Math. Ann. 101 (1929), 342-366. Corrigendum 103 (1930), 532.

[18] M. MORSE, Recurrent geodesics on a surface of negative curvature. Trans. Amer. Math. Soc. 22 (1921), 84-100.

[19] K. NishioKa, T.-A. TANAKA, Z.-Y. WEN, Substitution in two symbols and transcendence. Tokyo J. Math. 22 (1999), 127-136.

[20] G. PóLYA, Über gewisse notwendige Determinantenkriterien für die Fortsetzbarkeit einer Potenzreihe. Math. Ann. 99 (1928), 687-706.

[21] E. Prounet, Mémoire sur quelques relations entre les puissances des nombres. C. R. Acad. Sci. Paris 33 (1851), 225.

[22] M. QUEFFÉLEC, Transcendance des fractions continues de Thue-Morse. J. Number Theory 73 (1998), 201-211.

[23] D. Roy, Approximation simultanée d'un nombre et de son carré. C. R. Math. Acad. Sci. Paris 336 (2003), 1-6.

[24] D. RoY, Approximation to real numbers by cubic algebraic integers, I. Proc. London Math. Soc. 88 (2004), 42-62.

[25] A. Thue, Über unendliche Zeichenreihen. Norske vid. Selsk. Skr. Mat. Nat. Kl. 7 (1906), 1-22. Reprinted in Selected mathematical papers of Axel Thue. T. Nagell, ed., Universitetsforlaget, Oslo, 1977, pp. 139-158. 
[26] A. THUE, Über die gegenseitige Lage gleicher Teile gewisser Zeichenreihen. Norske vid. Selsk. Skr. Mat. Nat. Kl. 1 (1912), 1-67. Reprinted in Selected mathematical papers of Axel Thue. T. Nagell, ed., Universitetsforlaget, Oslo, 1977, pp. 413-478.

Jean-Paul Allouche

CNRS, LRI, Bâtiment 490

F-91405 Orsay Cedex, France

E-mail : allouche@lri.fr 